\title{
Self-Analysis: Do I Comprehend Case Series and Cohort Studies? A Fellow's Perspective
}

\author{
Daniel J. McCabe ${ }^{1,2}$ (D) \\ Received: 28 April 2019 / Accepted: 29 April 2019 / Published online: 7 May 2019 \\ (C) American College of Medical Toxicology 2019
}

Keywords Statistics $\cdot$ Observational Studies $\cdot$ Case Series $\cdot$ Cohort Studies

I have a confession: I struggle with the interpretation of observational studies. I have been involved in research since college and this has been a prolonged source of embarrassment. How could I not comprehend something as "simple" as an observational study? Since they are not complex prospective randomized-control trials, it was assumed observational studies should not be difficult to understand. As I progressed through residency, I read primary literature, listened to article descriptions on medical podcasts, attended the prestigious Emergency Medicine Basic Research Skills (EMBRS) workshop, and even published my own observational studies. But my confusion remained. Despite reading numerous articles to find a consistent definition, I could not find clarity.

Observational studies include case series, case-control studies, cross-sectional studies, longitudinal studies, and cohort studies. I found the differentiation of case series from cohort studies particularly confusing. In the classic textbook Designing Clinical Research by Hulley and Cummings, the authors dedicate an entire chapter defining the differences between cross-sectional and cohort studies. Simply, they state that cross-sectional studies can determine prevalence while cohort studies can determine rate of incidence. They explain that a cross-sectional study is performed at a single moment in time, whereas a cohort study is performed over time with a group that was "identified at the beginning of the study." In the entire book, the only mention of a case series was to explain that a cross-sectional study is impractical for rare disease and that a case series would be more appropriate, but no definition of a

Supervising Editor: Mark B. Mycyk, MD

Daniel J. McCabe

Daniel.J.McCabe@HealthPartners.com

1 Department of Emergency Medicine, Regions Hospital, 640 Jackson St, St Paul, MN 55101, USA

2 Minnesota Poison Control System, Minneapolis, MN, USA case series is ever provided [1]. How would one classify a study looking at a rare disease (or exposure), describing the effects (at a single moment or to a defined outcome), without a comparison group? It felt like everyone else understood the rules; but to me, the rules seemed to constantly change.

We cannot intentionally poison patients to answer important research questions. Even with prospective studies, it is impossible to control for exact dosing and time to presentation. As randomized trials of poisoned patients are difficult and arguably unethical to perform, we have to appreciate the nuance within our literature that mostly consists of uncontrolled observational studies. Not surprisingly, I realized early in fellowship that the interpretation of observational studies is an essential skill for our profession. The medical toxicology arena requires the rapid interpretation of observational studies to guide an enormous number of clinical decisions. Because of this, it was exciting when I was initially directed to the STROBE (Strengthening the Reporting of Observational Studies in the Epidemiology) statement [2], which is a checklist for authors to use prior to publication of observational studies. This guideline is commonly provided to authors by medical journals in their submission instructions. Theoretically, STROBE should give us all clarity on this subject. I read the STROBE statement multiple times, but still was left confused when trying to apply those guidelines to published case series and cohort studies. I decided to surrender on this matter and focus my efforts elsewhere; which (un)fortunately gave me solace.

As I was starting to gain confidence with primary literature interpretation, my boss recommended a paper for journal club. My heart sunk as I read the title "Distinguishing Case Series from Cohort Studies." [3] Immediately, I self-diagnosed imposter syndrome due to irritation, embarrassment, and plummeting confidence. Surprisingly, this article provided support for my frustration. The authors presented the following definitions for descriptive observational studies: A case series "is a study in which only patients with the outcome are 
sampled which does not permit calculation of an absolute risk." They contend a case series can be "incomplete" without consecutive study enrollment. A cohort study "should be based on exposure, follow-up is part of the study, and absolute risks can be calculated." In direct contrast to explanations by my previous teachers, in this paper Dekkers et al. state that a cohort study does not require a comparison group. They remind readers that the number of patients in the study should have no influence on the classification, and inadequate follow-up does not negate the cohort nature of the study. Maybe most importantly, they validated my concern regarding the absence of a case series description in the STROBE statement $[2,3]$. Moreover, Dekkers asserts that case series and cohort studies are frequently mischaracterized in published articles.

Another article written by Mathes clarifies and simplifies the differences between case series and cohort studies [3]. These authors agree that an observational study should be defined as a cohort study if a relative risk calculation can be determined from the data, even if the article's authors do not calculate the data themselves [3, 4]. These authors contend that in a case series, risk ratios cannot be calculated which means "a case series cannot be comparative." [4] Finally, I felt like I was starting to understand what everyone else already knew.

While attending a national toxicology meeting recently, I noticed some observational studies were actually misclassified. Some case series were labeled as cohorts, and some cohorts were really just case series. Such misclassification is not limited to the toxicology literature. Despite the STROBE statement that provides guidance on observational studies, at least one study demonstrated those guidelines are not widely followed [5]. Another study revealed miscategorization of $72 \%$ of the observational studies within the neurosurgical literature [6]. As a medical toxicologist I find this especially concerning in our own literature, because observational studies are major building blocks in systematic reviews as in the highly regarded EXTRIP papers. These systematic reviews may assign inaccurate quality of evidence to the initial studies. As evidence is cumulative, the initial evidence matters.

There are scant randomized-control trials examining the poisoned patient. Due to this, much of our clinical decisionmaking relies on individual observational studies and systematic reviews of observational studies. The designations of observational studies are important in the toxicology literature because they guide our clinical practice. As a cohort study is inherently given a higher level of evidence in a systematic review, an initial misclassification hinders any subsequent attempts at a higher level of evidence. As authors, reviewers, editors, and readers, we need to take ownership of the data we are provided. Authors of descriptive observational studies should be vigilant in providing all their raw data, especially if they are submitting a cohort study, to allow calculation of a risk. Authors of systematic reviews should analyze the observational studies to ensure they are properly classified. Peer review should place an increased emphasis on accurate labeling of observational studies. And after publication, readers should feel comfortable contacting a journal to express their concern if they find a categorization error. There will sometimes be differing interpretations, but an increased effort on classification is valuable. Because it appears I am not alone, I no longer find comfort in my confusion.

Now I have a new confession: I am skeptical of any article described as a case series or a cohort study unless I read it myself and understand the methods used. This is a small step in my academic progress, but sometimes the smallest steps are career changing.

Acknowledgements Special thanks to Dr. Trevonne Thompson for assistance with the production of this commentary.

Funding None

\section{Compliance with Ethical Standards}

Conflict of Interest None

\section{References}

1. Hulley SB, Cummings SR, Browner WS, Grady D, Newman TB. Designing clinical research. Philadelphia: Lippincott Williams \& Wilkins; 2013.

2. Von Elm E, Altman DG, Egger M, Pocock SJ, Gatzsche PC, Vandenbroucke JP. For the STROBE initiative. "The strengthening the reporting of observational studies in the epidemiology (STROBE) statement: guidelines for reporting observational studies". Lancet. 2007;370(9596):1453-7.

3. Dekkers OM, Egger M, Altman DG, Vandenbroucke JP. Distinguishing case series from cohort studies. Ann Intern Med. 2012;156(1 Pt 1):37-40.

4. Mathes T, Pieper D. Clarifying the distinction between case series and cohort studies in systematic reviews of comparative studies: potential impact on body of evidence and workload. BMC Med Res Methodol. 2017;17(1):107.

5. Karahalios A, Baglietto L, Carlin JB, English DR, Simpson JA. A review of the reporting and handling of missing data in cohort studies with repeated assessment of exposure measures. BMC Med Res Methodol. 2012;12:96.

6. Esene IN, Ngu J, Zoghby M, Solaroglu I, Sikod AM, Kotb A, et al. Case series and descriptive cohort studies in neurosurgery: the confusion and solution. Childs Nerv Syst. 2014;30(8):1321-32.

Publisher's Note Springer Nature remains neutral with regard to jurisdictional claims in published maps and institutional affiliations. 